\title{
X-ray phase contrast tomography from whole organ down to single cells
}

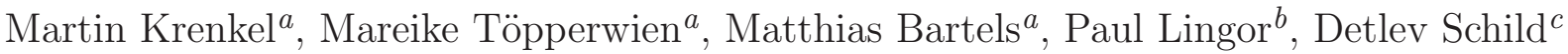 \\ and Tim Salditt ${ }^{a}$ \\ ${ }^{a}$ Institute for X-ray Physics, Georg-August-University Göttingen, Friedrich-Hund-Platz 1, \\ 37077 Göttingen, Germany \\ ${ }^{b}$ Department of Neurology, Georg-August-University Göttingen, University Medicine, \\ Robert-Koch-Str. 40, 37075 Göttingen, Germany \\ ${ }^{c}$ Department of Neurophysiology and Cellular Biophysics, Georg-August-University \\ Göttingen, Humboldtallee 23, 37073 Göttingen, Germany
}

\begin{abstract}
We use propagation based hard x-ray phase contrast tomography to explore the three dimensional structure of neuronal tissues from the organ down to sub-cellular level, based on combinations of synchrotron radiation and laboratory sources. To this end a laboratory based microfocus tomography setup has been built in which the geometry was optimized for phase contrast imaging and tomography. By utilizing phase retrieval algorithms, quantitative reconstructions can be obtained that enable automatic renderings without edge artifacts. A high brightness liquid metal microfocus x-ray source in combination with a high resolution detector yielding a resolution down to $1.5 \mu \mathrm{m}$. To extend the method to nanoscale resolution we use a divergent $\mathrm{x}$-ray waveguide beam geometry at the synchrotron. Thus, the magnification can be easily tuned by placing the sample at different defocus distances. Due to the small Fresnel numbers in this geometry the measured images are of holographic nature which poses a challenge in phase retrieval.
\end{abstract}

Keywords: X-ray, phase contrast, tomography, in-line holography, propagation based phase contrast, phase retrieval

\section{INTRODUCTION}

The three-dimensional structure of tissue of the central nervous system (CNS) is commonly assessed in form of thin sections in combination with histological staining and imaging techniques. A non-destructive survey of the small animal brain from the organ to the cellular level is to date beyond our capabilities, but extremely desirable for neuroscience, in particular if this could be combined with high throughput. X-ray imaging is a powerful technique to reveal internal structures of objects and tissues. With modern x-ray sources and optics, the imaging approach can be easily scaled over a wide range of resolutions, from the patient scale in the hospital down to nanometer resolution in x-ray microscopy. However, the biggest advantage of x-rays, i.e. their weak interaction and thus large penetration depth in tissue, can also be a significant disadvantage. Especially in case of imaging small structures and especially soft tissue, since low attenuation means low contrast, at least in conventional absorption imaging. One possibility to overcome this limitation is to use the stronger phase shift interaction of the object. The phase shift depends on the decrement $\delta$ in the complex refractive index $n=1-\delta+\mathrm{i} \beta$ which is up to 1000 times larger than $\beta$ for hard x-rays thus providing a huge potential contrast gain. There are several phase contrast x-ray imaging techniques which convert the phase shift, imposed by an object on the x-ray wave, into measurable intensity variations. At macroscopic resolution, suitable for medical imaging of entire organs, grating based interferometry provides

Further author information: (Send correspondence to M. K. or T. S.)

M.K.: mkrenke@gwdg.de

T. S.: tsaldit@gwdg.de 
a powerful approach to phase contrast imaging. ${ }^{1,2}$ For x-ray microscopy at the single cell level, Fresnel zone plate lenses with Zernike phase shifting rings are suitable in particular at low photon energy, where these optical elements have high efficiency. ${ }^{3}$ For hard x-rays, coherent scanning diffraction microscopy also yields the complex valued object transmission function which contains absorption and phase information. ${ }^{4,5}$ For intermediate and scalable resolution and field of view, propagation based phase contrast as described in?,6-8 offers great potential. The potential of novel coherent x-ray imaging methods for neuroscience has recently been reviewed. ${ }^{9}$

In this paper we use propagation based phase contrast tomography to image soft biological tissue of the CNS in mouse and Xenopus laevis tadpoles, in order to provide a benchmark and proof-of-concept study. With regard to previous work, we mainly explore two directions: (A) The use of compact laboratory microfocus x-ray sources as a tool for neuro scientists circumvents the need for synchrotron radiation, which is considered to be less accessible. To realize tomography of CNS tissue at microfocus source, we employ novel liquid jet high brightness anodes, ${ }^{10}$ a range of state-of-the art detectors, and an optimized imaging geometry. ${ }^{11}$ (B) Tomography of unstained CNS tissue in the hydrated state. Contrast of soft tissue can be generated based on the mentioned phase shift of the x-ray wave with respect to the empty beam going through air. However, in the hydrated state, it is not the phase shift of tissue with respect to air, but the much weaker difference to water. Without any labeling with contrast agents, the native internal density distribution can be used for contrast formation. Today most synchrotron setups use propagation based phase contrast only in the so called direct contrast regime, ${ }^{?, 12}$ where the potential of the phase shift is not fully exploited. However, in the holographic regime more details can be observed due to a better contrast transfer. As the observed images do not show the structures of interest but a holographic version of it, phase retrieval is an indispensable step in this regime but a high quality illumination is needed to circumvent reconstruction artifacts. To this end we use x-ray waveguide optics in this work as a highly controlled secondary source for imaging.? Apart from exceptional spatial coherence, x-ray waveguides also provide small source sizes down to below $10 \mathrm{~nm}$ and wave fronts of high quality (low aberrations), meeting the idealized requirements of perfect illumination by a plane or spherical wave, which is ubiquitous in propagation imaging. The next two sections start with a brief discussion of suitable phase retrieval algorithms followed by a demonstration of the experimental applications to CNS tissue.

\section{IMAGING MODES AND PHASE RETRIEVAL}

\subsection{Propagation based phase contrast}

By free space propagation, structural information of an object, encoded in the phase of the exit wave, is transformed to a measurable intensity distribution as the wave perturbed by the object interferes with itself. The starting point to understand the image formation in this so called propagation based phase contrast method and the corresponding phase retrieval, is the wave equation. For hard x-rays, the paraxial scalar wave equation provides a suitable framework, if polarization effects and scattering to high angles can be neglected. This approximation is justified if the interaction of the object can be described on length scales much larger than the x-ray wavelength $\lambda$, i.e. if a continuous and slowly varying index of refraction $n(\mathbf{r})=1-\delta(\mathbf{r})-\beta(\mathbf{r})$ is a suitable description for the object. A solution for the propagated exit wave described by the paraxial (parabolic) wave equation can then be derived by the angular spectrum method which leads to the following expression describing the evolution of a wave in free space ${ }^{13}$

$$
\psi\left(\mathbf{r}_{\perp}, z\right)=e^{\mathrm{i} k z} \mathcal{F}_{\perp}^{-1}\left[\mathrm{e}^{-\mathrm{i} z / 2 k\left(\xi_{x}^{2}+\xi_{y}^{2}\right)} \mathcal{F}_{\perp}\left(\psi\left(\mathbf{r}_{\perp}, 0\right)\right)\right]
$$

Here $\psi\left(\mathbf{r}_{\perp}, z\right)$ denotes the complex valued wavefunction in a plane with coordinate vector $\mathbf{r}_{\perp}=(x, y)$ perpendicular to the optical axis at a distance $z$ along the optical axis. $\psi\left(\mathbf{r}_{\perp}, 0\right)$ is the initial wave field, e.g. in the exit plane of the object and $k=2 \pi / \lambda$ is the wavenumber. $\mathcal{F}_{\perp}$ denotes the Fourier transform in the lateral coordinates $\xi_{x}$ and $\xi_{y}$ that in the experiment are expressed in units of the pixel sizes $p_{x}$ and $p_{y}$. Note that the experimental length scales enter the equations in a certain combination so that they can be 
reduced to a single unitless parameter describing the image formation. This so called Fresnel number can be written as

$$
F_{x / y}=\frac{p_{x / y}^{2}}{\lambda \cdot z}
$$

As in most cases the pixel size $p_{x}$ equals $p_{y}$, we neglect the subscript in the following for $p$ and $F$. For very small propagation distances $z$ or correspondingly very large Fresnel numbers $F$, the propagation has not yet influenced the initial wave-function, which corresponds to the regime of classical geometric optics without diffraction. Contrarily, for very small Fresnel numbers $F \ll 1 / N^{2}$ with $N$ the number of pixels in the image, the propagation can be described as a Fourier transformation (Fraunhofer regime) whereas for Fresnel numbers in the range of $F \approx 1$, the resulting wave is in the Fresnel nearfield regime. Depending on the actual value of the Fresnel number, the measured intensity distribution directly reflects the projected structure of the object (direct contrast regime) or is already significantly affected by interference leading to holographic intensity oscillations, observed for smaller Fresnel numbers (intermediate regime).

\subsection{Phase retrieval}

To obtain a quantitative image of the object from measured holographic intensity distributions, different phase retrieval algorithms are employed. As for the image formation, only the Fresnel number $F$ is needed as a parameter to reconstruct the image, the combination of $\lambda, z$ and $p$ in terms of $F$ is sufficient. In this work we use two different phase retrieval algorithms for different imaging regimes.

\subsubsection{Bronnikov Aided Correction}

One of the two phase retrieval algorithms used below is denoted as Bronnikov Aided Correction (BAC). It is based on a linearization of the transport of intensity equation (TIE) ${ }^{14,15}$ and is valid for weakly absorbing objects and small propagation distances, thus large Fresnel numbers. In this so called direct contrast regime, the phase contrast image will show bright and dark fringes especially around edges of the object, as given by the two-dimensional (2D) Laplacian of the projected phase. Under these assumptions, a simplified form of the TIE can be written in the following form

$$
I\left(\mathbf{r}_{\perp}, \Delta z\right)=I_{0}\left(1-\frac{\Delta z}{k} \nabla_{\perp}^{2} \phi(x, y)\right) .
$$

Here $I_{0}$ describes the intensity distribution in the object exit plane and $I\left(\mathbf{r}_{\perp}, \Delta z\right)$ is the intensity in a plane located at $\Delta z$ behind the sample in the detection plane. For a pure phase object under homogenous illumination, $I_{0}$ is constant. However for a weakly absorbing object, this equation is to some extent still valid and $I_{0}$ can be regarded as a pure absorption image which could be measured directly behind the object (if experimentally possible) and which is to be reconstructed by the BAC algorithm. To this end a first guess for the object's phase distribution is derived from the measured image by inverting Eq. (3) with Fourier methods, yielding

$$
\phi(x, y)=2 \pi F \mathcal{F}_{\perp}^{-1}\left(\frac{\mathcal{F}_{\perp}\left(I / I_{0}-1\right)}{\left|\xi_{\perp}^{0}\right|^{2}+\alpha}\right) .
$$

Here $\xi_{\perp}^{0}=p \cdot\left(\xi_{x}, \xi_{y}\right)$ denotes the unitless reciprocal variable which eliminates the pixel size dependence. Thus the equation only depends on the Fresnel-number $F$. As for $\xi_{\perp}^{0} \rightarrow 0$ the denominator diverges, a regularization parameter $\alpha$ is added to compensate for low frequency parts in the image which result from absorption. Subsequently a hypothetical pure phase image $C=1-\frac{\Delta \mathrm{Z}}{k} \nabla_{\perp}^{2} \phi$ of the object is obtained. The measured intensities are then divided by this image $C$, yielding

$$
I_{a}(x, y)=\frac{I\left(\mathbf{r}_{\perp}, \Delta z\right)}{1-\gamma \nabla_{\perp}^{2} \phi(x, y)},
$$

to derive the absorption imaged of the sample. As the regularization parameter $\alpha$ is chosen based on visual inspection of the image, the factor $\Delta z / k$ in the experiment may lead to wrong results. Thus it is replaced by 
an effective parameter $\gamma$ which will be also chosen based on visual inspection. The full reconstruction thus works as follows: First the phase image $\phi(x, y)$ is reconstructed by choosing $\alpha$ such that edge enhancement effects are compensated without blurring the image too much. Afterwards the pure absorption image $I_{a}(x, y)$ is obtained by tuning $\gamma$ to reduce edge enhancement without inverting it. This kind of phase retrieval is valid if the image shows just weak and homogenous absorption and is recorded in the direct contrast regime. The advantage is that it delivers significantly sharper reconstructions compared to just using Eq. (4). ${ }^{14}$

\subsubsection{Contrast Transfer Function}

For smaller Fresnel-numbers $F$ the image will not just show edge enhancement effects, but multiple fringes will appear and the image loses resemblance to the real structure. In fact, an in-line hologram of the object is recorded as originally proposed for electron beam holography by Gabor. ${ }^{16}$ In this imaging regime, phase retrieval is not only needed to get quantitative contrast values from the measured images but also to reconstruct in particular high frequency spatial information, which has been blurred by diffraction. One deterministic method, which is very robust to experimental imperfections and noise, is based on the contrast transfer function $(\mathrm{CTF})$. It can be derived by again assuming a weakly absorbing object and a slowly varying phase but making no assumption to the propagation distance. ${ }^{17-19}$ Writing the complex valued field in dependence of the attenuation $\mu$ and phase distribution $\phi$ of the object and using Eq. (1) the CTF can be derived by a first order expansion

$$
\tilde{I}\left(k_{x}, k_{y}, z\right)=\delta_{D}+2 \tilde{\phi} \sin \chi-\tilde{\mu} \cos \chi
$$

Here $\tilde{I}=\mathcal{F}_{\perp}(I(x, y, z))$ denotes the lateral Fourier transform of the intensity in a plane with distance $z$ from the object exit plane, $\delta_{D}$ is the Dirac-delta-distribution and $\phi=k z \bar{\delta}$ and $\mu=2 k \bar{\beta}$ are the projected phase and attenuation. The ${ }^{\sim}$ symbolizes the Fourier transformation and $\chi=z /(2 k)\left(\xi_{x}^{2}+\xi_{y}^{2}\right)=4 \pi / F\left(\left|\xi_{\perp}^{0}\right|^{2}\right)$ is a propagation term which again only depends on $F$ if unitless Fourier coordinates are used. By introducing an error metric for $n$ images $S=\sum_{n}\left\|\tilde{I}_{\text {exp,n }}-\tilde{I}\left(k_{x}, k_{y}, z\right)\right\|^{2}$, using Eq. (6) for $\tilde{I}$ and minimizing $S$ with respect to $\tilde{\phi}$, one can easily derive a reconstruction formula, e.g. for a pure phase object $(\mu=0)$

$$
\tilde{\phi}=\frac{\sum_{n} \tilde{I}_{e x p, n} \sin \chi_{n}}{\sum_{n} \sin ^{2} \chi_{n}+\alpha} .
$$

The additional, frequency dependent regularization parameter $\alpha$ is introduced, to compensate zeros of the denominator. This regularization parameter can also be split up into two in order to treat high and low spatial frequencies differently. Experimental data recorded in a cone beam geometry also needs additional imaging processing steps: each frame has to be resized for common overlap and magnification, requiring careful aligned images between frames recorded at different $z$. We do this by first reconstructing a coarse phase distribution based on a single distance reconstruction $(n=1)$ of the resized holograms and using the algorithm presented in ${ }^{20}$ to register the images for different defocus distances.

\section{EXPERIMENTAL REALIZATION}

\subsection{Sample preparation}

\subsubsection{Supercritical dried tadpole heads}

For the laboratory experiments, the olfactory system of Xenopus laevis tadpoles of about stage $54^{21}$ are used as they are a well known model organism for neuronal studies concerning the olfactory system. ${ }^{22}$ As the contrast of soft tissue to water is rather weak, dried tadpole heads were used. To preserve the ultra structure of the samples and prevent it from shrinking the samples are supercritically dried. The finally dried heads are mounted to a sample holder. A photograph of the final tadpole head specimen is shown in Fig. 2 (a). The dashed rectangle indicated the position used for the tomographic measurements. 


\begin{tabular}{llllll}
\hline & Fig. (2) (b) & Fig. (2) (c) / Fig. (3) & Fig. (4) & Fig. (5) & Fig. (6) \\
\hline \hline exposure time & $15 \mathrm{~s}$ & $20 \mathrm{~s}$ & $120 \mathrm{~s}$ & $2.5 \mathrm{~s}$ & $100 \mathrm{~s}$ \\
camera & FDI & LCX & PCO & sCMOS & $\mathrm{sCMOS}$ \\
source power & $40 \mathrm{~W}$ & $4 \mathrm{~W}$ & $40 \mathrm{~W}$ & $5 \cdot 10^{8} \mathrm{ph} / \mathrm{s}$ & $5 \cdot 10^{8} \mathrm{ph} / \mathrm{s}$ \\
resolution limit & $3 \mu \mathrm{m}$ & $3 \mu \mathrm{m}$ & $1.5 \mu \mathrm{m}$ & $25 \mathrm{~nm}$ & $25 \mathrm{~nm}$ \\
energy & $9.25 \mathrm{keV}$ & $17.5 \mathrm{keV}$ & $9.25 \mathrm{keV}$ & $13.8 \mathrm{keV}$ & $13.8 \mathrm{keV}$ \\
number of projections & 1 & 1830 & 915 & 720 & 1 \\
number of distances & 1 & 1 & 1 & 3 & 8 \\
effective pixel size & $4.25 \mu \mathrm{m}$ & $2.6 \mu \mathrm{m}$ & $0.6 \mu \mathrm{m}$ & $193 \mathrm{~nm}$ & $25 \mathrm{~nm}$ \\
Fresnel number & 2.1 & 0.9 & 0.2 & $2.9 \cdot 10^{-3}$ & $3.6 \cdot 10^{-4}$ \\
\hline
\end{tabular}

Table 1. Experimental parameters used for the images shown. Exposure time is the total exposure time per distance per projection. The different cameras are described in the main text. Source power shows the electron-beam power for the laboratory source and the number of photons per second exiting the waveguide for the synchrotron experiments. The resolution limit is given either by the source size $(3 \mu \mathrm{m})$, by the detector resolution $(1.5 \mu \mathrm{m})$ in the inverse geometry, or by the effective source size of the x-ray waveguide $(25 \mathrm{~nm})$. All resolution values are determined from measurements on x-ray test patterns. The listed energy is the K-alpha line for the laboratory experiments, which contributes about half to the total spectrum. At the synchrotron the energy is monochromatic. The Fresnel number is calculated for a structure of one pixel at the peak energy.

\subsubsection{Brain slice}

For synchrotron experiments it is also possible to investigate hydrated samples as the illuminating beam has a higher coherence and a much smaller bandwidth, thus enabling the increase of contrast by choosing smaller Fresnel numbers. As a proof of concept a thick slice of a mouse brain was prepared and mounted in a liquid chamber. A wild type mouse was sacrificed with $\mathrm{CO} 2$ and its brain perfused with $4 \%$ para-formaldehyde (PFA) to fix the tissue. Afterwards the brain was dissected and fixed in PFA over night. The fully fixed brain was cut into $1 \mathrm{~mm}$ thick slices, which were stored in phosphate buffered solution (PBS) until the experiment. Directly before the measurements one slice was mounted in PBS between two polypropylene foils on aluminum rings forming a liquid chamber. ${ }^{23}$ The rings were glued together and mounted on a sample holder. Figure 5 (b) shows a photograph of a final preparation with the brain slice kept in PBS between the two rings.

\subsubsection{Single neuronal cells}

In addition to the fixed brain slices, also primary neurons from cell culture have been examined. To this end, the hippocampus from 18 days old rat embryos was extracted. By adding trypsin for the digestion of the tissue and DNAse to dissolve the thereby released DNA, the neurons can be extracted. The digestion of the tissue is stopped by adding bovine serum. These neurons are then given onto a silicon nitride membrane which was sterilized before. For better adhesion of the neurons, the membrane was coated in Poly-L-ornithine solution for about four hours. Growth of the neuron cell culture was enabled by incubating the membrane in medium for one week. As a final step of the preparation, the cells were fixed with PFA and stored in PBS. Prior to the measurements, a second silicon nitride membrane is glued onto the first one to create a closed liquid chamber which is then mounted on a sample holder.

\subsection{Laboratory phase contrast imaging with microfocus x-ray sources}

By using microfocus sources together with an optimized geometry and high resolution detectors, $\mathrm{x}$-ray phase contrast imaging can be realized in the laboratory. Here we use a liquid metal jet x-ray source (Excillum) which allows an up to ten times higher flux density for small source sizes. ${ }^{24} \mathrm{~A}$ metal alloy based on gallium, indium and tin (Galinstan) which is liquid at room temperature is used for broadband x-ray emission with a peak at the K-alpha line of gallium at $9.25 \mathrm{keV}$. Additionally, a solid molybdenum target can be used for the measurements with a peak energy at $17.5 \mathrm{keV}$. 
(a)

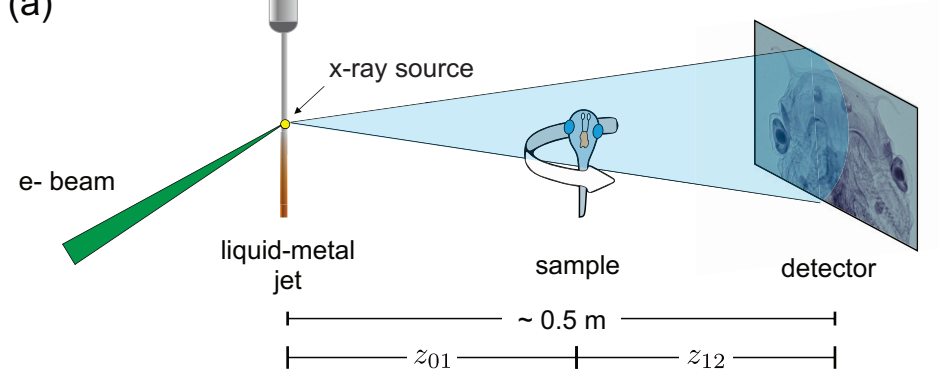

(b)

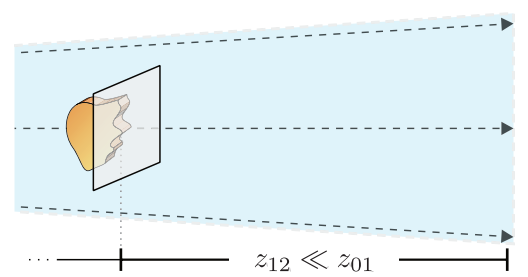

(c)

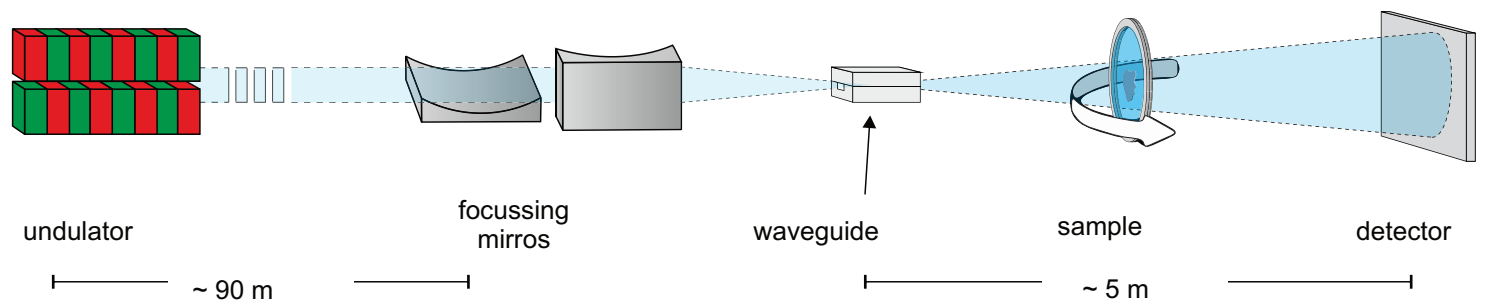

Figure 1. (Color online) Schematics of the different experimental geometries used in this work. (a) Laboratory setup with a high brightness microfocus source based on a liquid metal jet. Accelerated electrons are hitting the liquid metal target in a spot of around $10 \mu \mathrm{m}$ in which the x-rays are created. The resulting divergent beam is used for imaging either in (a) a magnifying mode with the sample positioned close to the source or in (b) an "inverse geometry" with the sample close to the detector. In (a), geometric magnification by the divergent beam is exploited, resulting in a resolution which is then limited by the (effective) source size. Contrarily, in the quasi parallel beam case of (b), the pixel size of a high-resolution detector limits the resolution. In this mode, a resolution down to about $1 \mathrm{\mu m}$ is possible even at the laboratory source. (c) Synchrotron setup used for the waveguide based imaging. The prefocused x-rays are filtered by an x-ray waveguide resulting in a very coherent beam consisting of only a few optical modes with an effective source size of below $20 \mathrm{~nm}$. The resulting divergent beam can be used for high resolution imaging.

\subsubsection{Magnifying geometry}

Figure 1 (a) shows the schematic of a magnifying imaging mode. This "classical" mode is based on using an efficient x-ray detector with pixels larger than the desired resolution and employing the effect of geometric magnification. Thus zooming is easily realized by moving the sample to different positions in the divergent beam. Two different detectors are used in this mode. The first one is a camera in which a Gadox scintillator is coupled by a fiber optic plate to a CCD camera (FDI, Photonic Science) with a pixel size of $6.54 \mu \mathrm{m}$. The second detector is a directly illuminated CCD camera with $20 \mu \mathrm{m}$ pixel size (LCX, Princeton Instruments). The x-ray source size limits the resolution in this mode, which is in the range of about 3 to $4 \mu \mathrm{m}$ as confirmed by a resolution test pattern. Figure 2 (b) and (c) show exemplary phase contrast projections of a dried tadpole head measured at two different magnifications with the different detectors, showing the entire head in Fig 2 (b) and a smaller region of interest (ROI) with the olfactory system in Fig. 2 (c). In both cases the edges of the image are enhanced by interference effects improving the contrast of the soft tissue. The relevant experimental parameters are listed in Tab. 1. By rotating the sample and recording images for several angles in the range of 0 to 180 degree, a tomographic dataset can be recorded. This is done in the configuration shown in Fig. 2 (c).

Figure 3 shows the results of a tomographic scan. The tomographic reconstruction is performed with a fast cone beam reconstruction software (Bronnikov Algorithms) based on filtered back projection (FBP). In Fig. 3 (a) an orthoslice perpendicular to the rotation axis is shown in which no phase retrieval was performed and just the empty beam-corrected intensity distributions were used for the reconstructions. A 

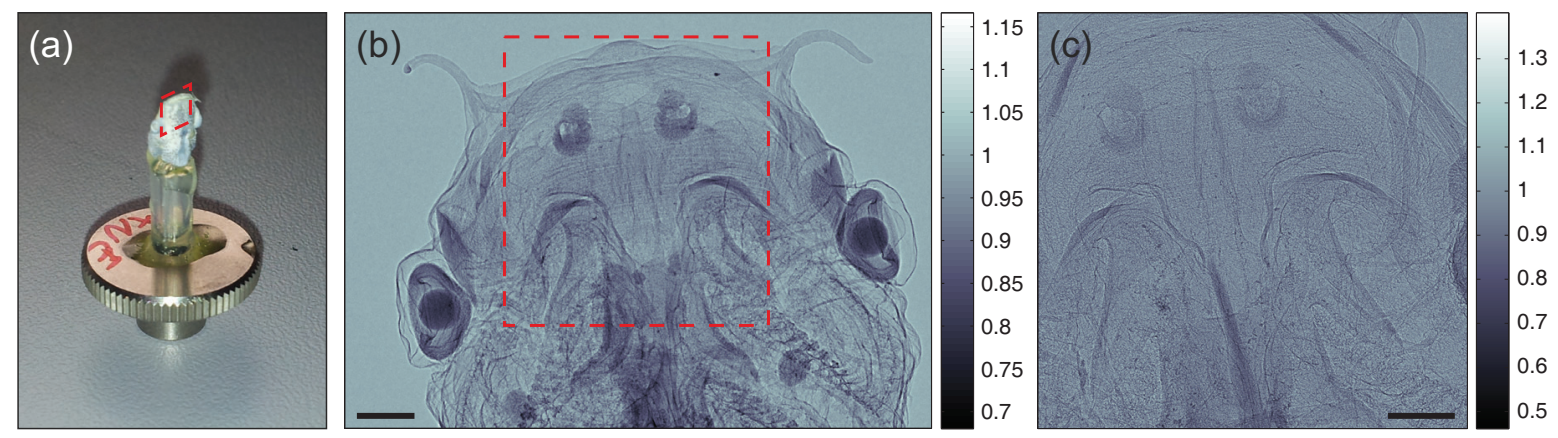

Figure 2. (Color online) Phase contrast measurements recorded in magnifying mode in the laboratory. (a) Photograph of a supercritical dried tadpole head mounted on a sample holder (b) Phase contrast projection image of the tadpole head. The dashed rectangle indicates the region used for the tomographic measurements which cover the olfactory system. For the measurements, the magnification was increased by moving the sample closer to the source. (c) One exemplary projection of the tomographic scan measured at the position indicated in (b). The corresponding results are shown in Fig. 3. Scalebars denote $500 \mu \mathrm{m}$.

profile through the dashed blue line is shown in (b), reflecting the internal structure, in particular interfaces by the edge enhancement inherent in direct contrast images. Figure 3 (c) shows the same slice but with BAC phase retrieval prior to the tomographic reconstructions. A profile through the same line as in (a) is shown in Fig 3 (d). It can be clearly seen that regions in which denser tissue is located have a different density value over the whole range, and that not just edges are visible. This enables automatic three dimensional (3D) renderings of the structure by assigning a different color and transparency for every density value. Such a $3 \mathrm{D}$ volume rendering is shown in Fig. 3 (e) in which some structures of the olfactory system are manually segmented for better recognition. Neuronally relevant tissue can be identified in 3D, demonstrating that the method can be implemented at laboratory sources and used to address 3D histological and micro anatomical questions in neuroscience.

\subsubsection{Inverse geometry}

To further increase the resolution, a different experimental geometry can be used for phase contrast imaging. ? Figure 1 (b) schematically shows the arrangement of sample and detector in this "inverse" geometry. By moving the sample rather close to the detector, the resolution is limited mainly by the detector. To this end, a very high resolution detector is used which is based on a $20 \mu \mathrm{m}$ thin single crystal LuAG:Ce scintillator (Crytur) which is viewed by an optical microscope consisting of a 10-fold magnification and a cooled CCD camera (pco.2000, PCO). This kind of detector is usually used at synchrotron sources where a resolution down to below $1 \mu \mathrm{m}$ is possible. ${ }^{25}$ The optical system behind the scintillator transmits only a small part of the generated light to the camera, but the high flux provided by the liquid metal source enables the use of this kind of detectors also in the laboratory with reasonable exposure times. With the detector used here, a resolution of about $1.5 \mu \mathrm{m}$ could be achieved. Due to the high resolution small Fresnel numbers and thus phase contrast images can be recorded even for rather short propagation distances in a range of e.g. $20 \mathrm{~mm}$ between object and detector.

Figure 4 (a) shows a phase contrast image of a ROI within the tadpole head showing the olfactory system at a higher resolution. In this imaging mode a further tomographic scan was recorded. A list of the parameters used in the measurements is shown in Tab. 1. Phase retrieval is again performed with the BAC algorithm as the images are still in the direct contrast regime. Figure 4 (b) shows an orthoslice perpendicular to the rotation axis in which a similar structure as in Fig. 3 can be observed. In Fig. 4 (c) a $3 \mathrm{D}$ rendering consisting of three perpendicular orthoslices together with a segmented olfactory nerve (ON) is shown. The higher resolution enables the representation of relevant internal tissue structure, e.g. of the transition from the olfactory nerve to the olfactory bulb $(\mathrm{OB})$ in more detail. 

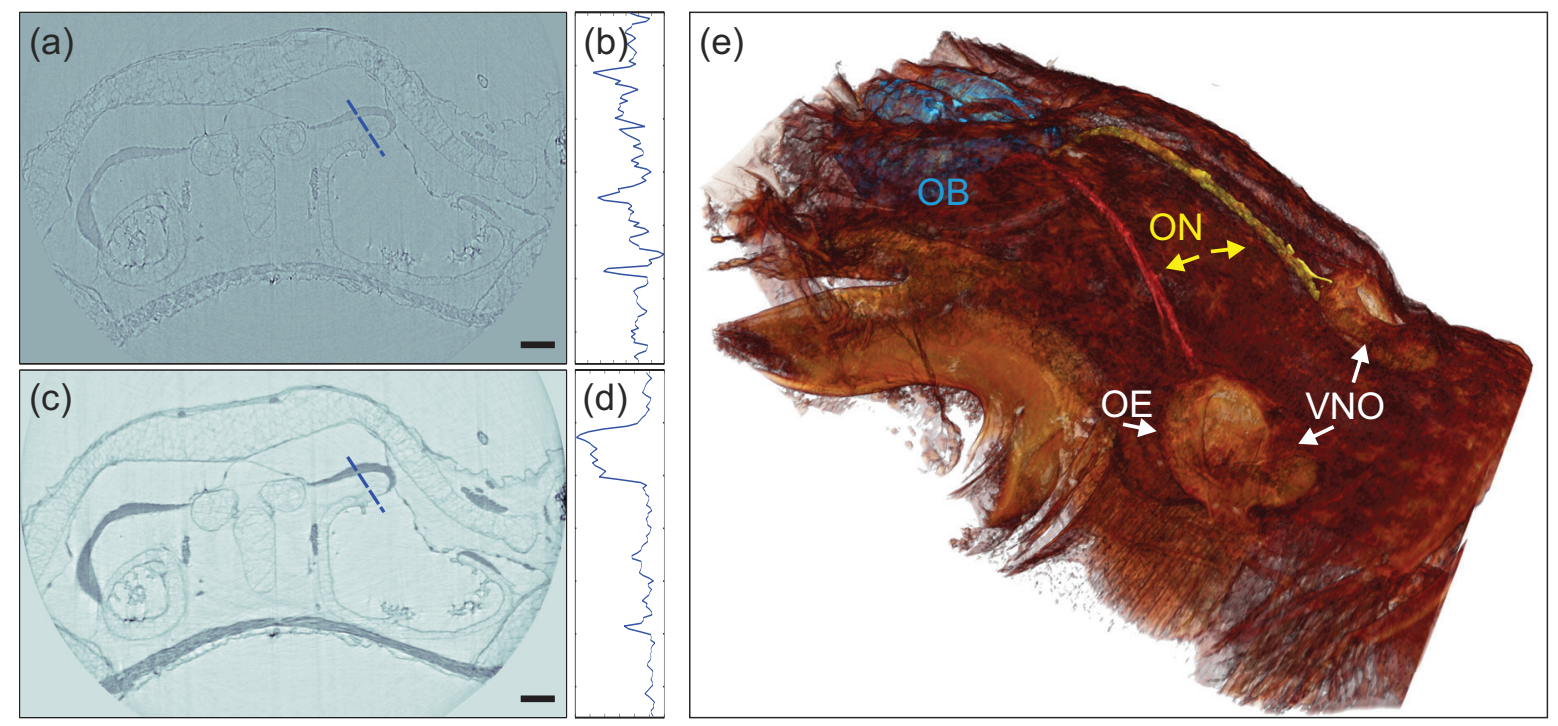

Figure 3. (Color online) Tomographic reconstruction from data recorded with the laboratory setup (magnifying mode). (a) FBP reconstructed slice of the measured (raw) phase contrast projections without phase retrieval, and (c) the same after phase retrieval by the BAC algorithm, along with the respective line cuts in (b) and (d) through the same image detail. In (a) the internal structure is visible due to the edge enhancement inside the tissue, as indicated by (b) the profile along the dashed line, but the lack of phase retrieval leads to artifacts, while the BAC + FBP reconstruction in (c) with the corresponding line cut (d) shows that the edge enhancement has been successfully inverted to realistic contrast levels. (e) 3D rendering based on an automatic volume rendering of different density values. The vomeronasal organs (VNO) and the olfactory epithelia (OE) can be clearly identified. Additionally the olfactory nerves $(\mathrm{ON})$ are manually segmented in red / yellow and the olfactory bulb (OB) of the brain is labeled in blue. Scalebars denote $200 \mu \mathrm{m}$.

\subsection{X-ray waveguide imaging for high resolution phase contrast tomography}

The "inverse geometry" is widely used in synchrotron imaging as it can be used without any optics. Moreover, the low divergence of synchrotron beams allows for a large range of propagation distances to choose the Fresnel number according to the contrast of the sample. For the high resolution detectors described before the resolution is limited by the diffraction limit of the visible light created in the scintillator, thus being in the range of about 300 to $500 \mathrm{~nm}$. However, the detection efficiency in this scheme is rather low.

Another promising approach is to again use a magnifying setup in which the resolution is limited by the (effective) source size. As the synchrotron radiation is very brilliant, it can be focused to small spots with large flux densities. This enables the use of x-ray waveguides (WG) consisting of low density guiding channels and a high density cladding, which have to be precisely aligned in a focused beam. The general idea is sketched in Fig. 1 (c). Here we have used a crossed multilayer WG with $59 \mathrm{~nm}$ guiding layer over a length of about $0.7 \mathrm{~mm}$ as the illumination forming optic. Waveguides of this kind are described in detail e.g. by Krüger et. al. ? Based on total reflection of the x-rays inside these channels, the radiation is confined and it can be shown that only a few optical modes can propagate through the channels. This increases the spatial coherence, ${ }^{26}$ significantly improves the quality of the illumination wave front ${ }^{27}$ and leads to a smaller effective source size. The WG used here produces a source size of $16 \mathrm{~nm}$ according to finite difference simulations which is confirmed by reconstructions of the measured farfield intensity distribution. Using this WG, resolution values below $30 \mathrm{~nm}$ were achieved previously, as demonstrated by two-dimensional test patterns. ${ }^{27}$

Figure 5 shows the results obtained for a hydrated thick brain slice imaged in an x-ray waveguide setup. In Fig. 5 (a) an empty beam-corrected phase contrast projection is shown, which is not in the direct contrast regime anymore. Hence the CTF-based phase retrieval algorithm described above is used. To 

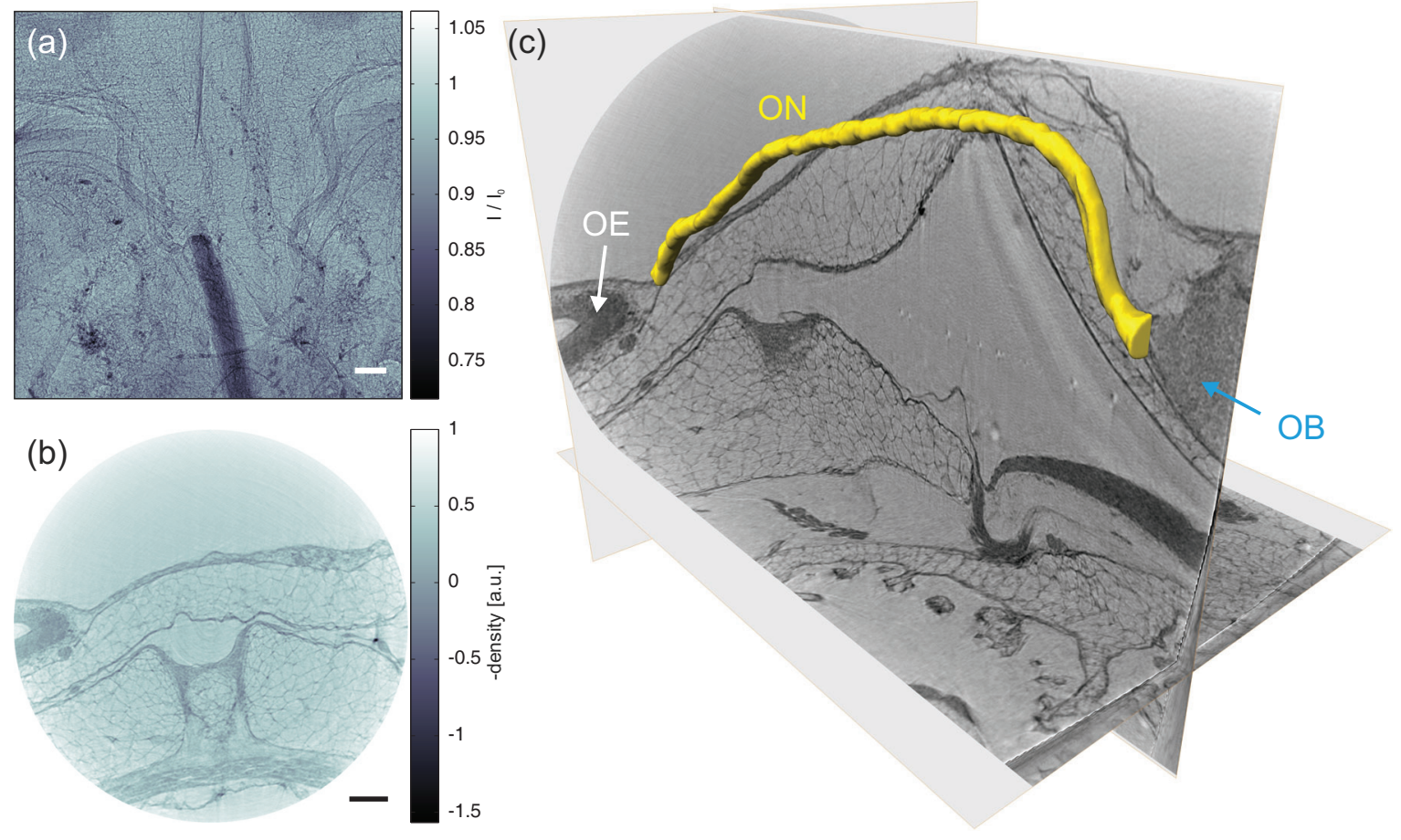

Figure 4. (Color online) Phase contrast tomography with the high resolution "inverse geometry" mode. (a) Phase contrast projection of the olfactory system recorded with a high resolution detector at a liquid-metal x-ray source. (b) Reconstructed slice (BAC phase retrieval + FBP) of a corresponding tomographic measurement. (c) 3D Rendering showing three perpendicular orthoslices together with a manually segmented olfactory nerve (ON). Scalebars denote $100 \mathrm{\mu m}$.

this end, the intensity distribution is measured in three planes with slightly different Fresnel numbers by moving the sample in the divergent beam. The corresponding reconstructed projected phase distribution is shown in Fig. 5 (d). It can be clearly seen that edges and small features inside the projections appear sharper after the reconstruction. However, as many structures inside the $1 \mathrm{~mm}$ thick slice are overlayed, a tomographic reconstruction in this setting is necessary to better distinguish features of the internal structure. The tomographic reconstruction is performed by using a standard filtered backprojection (FBP) algorithm implemented in Matlab as cone beam effects are negligible for the low divergence angles present at the synchrotron. Detailed experimental parameters are listed in Tab. 1. Figure 5 (e) shows a reconstructed orthoslice parallel to the rotation axis and in Fig. 5 (f) two perpendicular orthoslices are shown, which show that the observed structures are present in 3D. Unfortunately the slice perpendicular to the rotation axis shows some artifacts due to a non perfect circular movement. The rotational movement may be disturbed by an overlayed movement of the sample. A border between tissue with a higher cell density and a probably water-filled area can however be clearly recognized. Several dark spots, corresponding to a higher density can also be clearly identified. We attribute this spots to the nuclei of single cells. Note that these nuclei are visible without any staining of the tissue. To locate the position of the measured tomogram inside the whole brain slice, we scanned the sample laterally to produce a $2 \mathrm{D}$ overview. Based on this scan a sketch indicating the position is created, which is shown in Fig. 5 (c).

To prove that single neuronal cells can be resolved in the hydrated and unstained state, neurons were imaged in a wet chamber using the same waveguide configuration as presented above. Figure 6 (a) shows an optical phase contrast micrograph of a single neuron. The dashed area denotes the area which was then imaged using the x-rays. In (b) an empty beam-corrected phase contrast projection is shown which appears very holographic due to the small Fresnel number present at this magnification. By recording a total of 

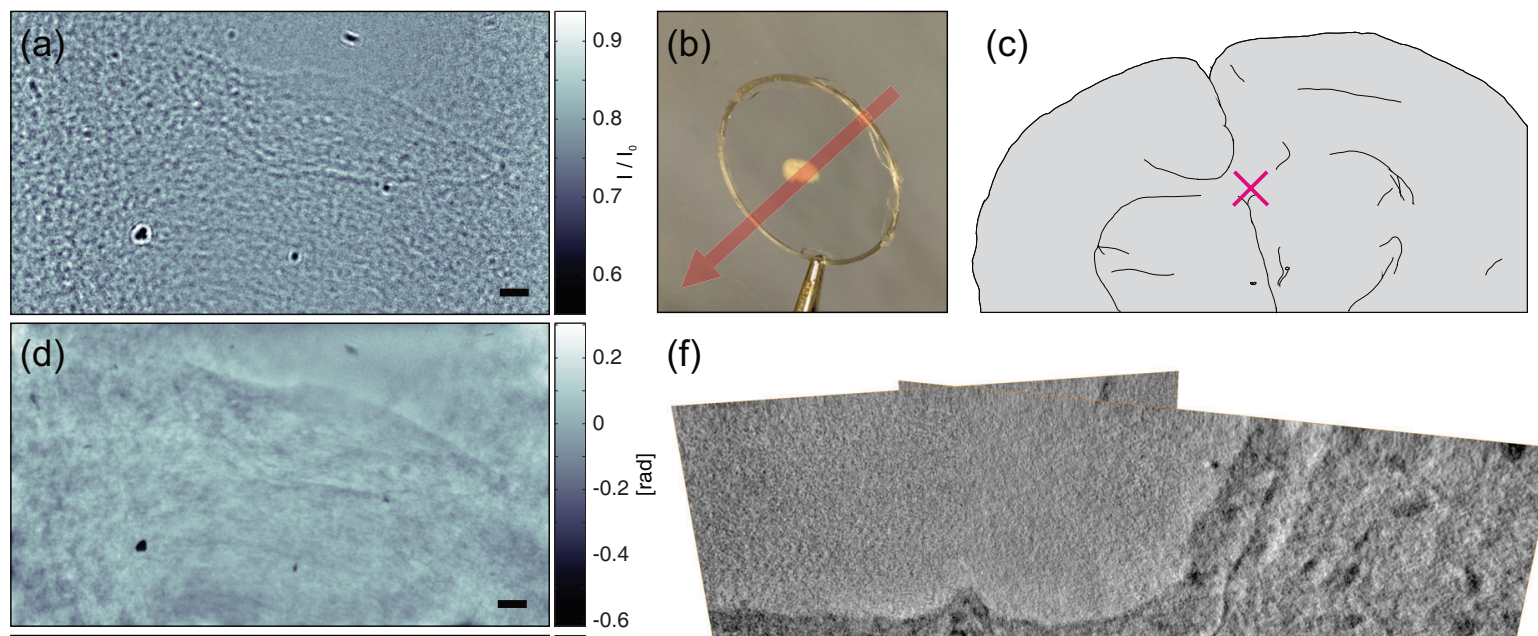

(f)

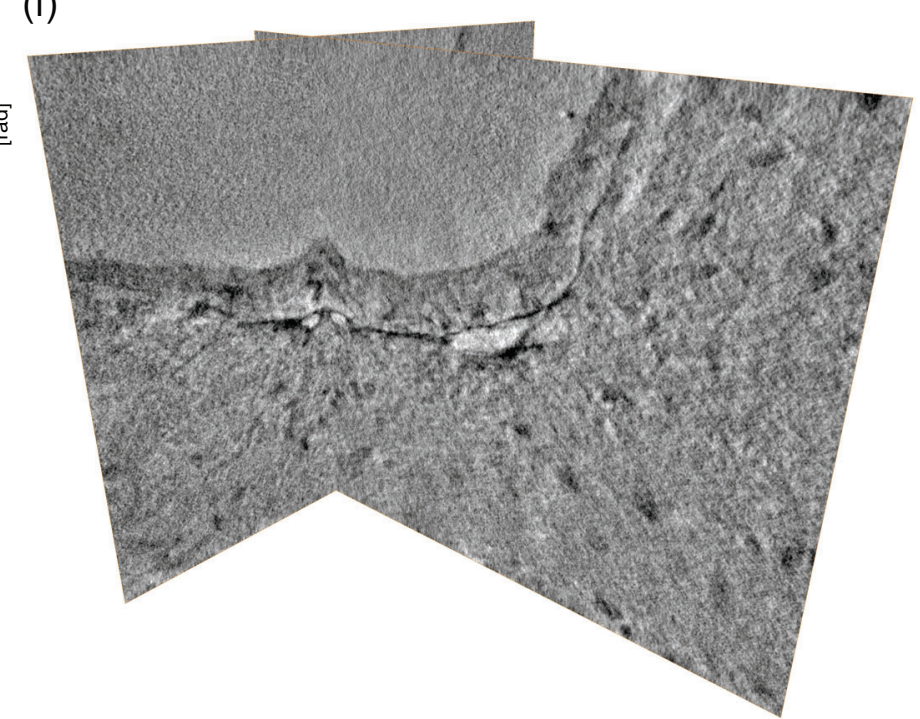

Figure 5. (Color online) Phase contrast tomography of hydrated and unstained CNS tissue recorded in magnifying geometry using synchrotron radiation and waveguide optics. (a) Phase contrast projection of a small part inside the brain. Due to the smaller Fresnel numbers the structure appears holographic, thus several fringes are present around every structure. (b) Photograph of the mounted brain slice. The red arrow indicates the direction of the x-ray beam. (c) Orientation sketch based on raster scanning the sample, indicating the position used for the tomographic measurements. (d) CTF retrieved phase distribution obtained using three distances (see main text). (e) Cut through the tomographic reconstruction of the brain slice, showing the internal tissue structure. Single nuclei of cells can be resolved as indicated e.g. by the arrow. (f) $3 \mathrm{D}$ representation of two perpendicular slices. Scalebars denote $20 \mu \mathrm{m}$.

eight projections for different Fresnel numbers, a CTF-based phase reconstruction, shown in Fig 6 (c) can be obtained. The structure clearly corresponds to what can be seen in the optical micrograph and even some parts of the axons can be identified. Note that the total dose used to obtain the $2 \mathrm{D}$ reconstructed phase in Fig. 6 (c) is more than eight times larger than the total dose used for the tomographic scan in Fig. 5. Thus it can be concluded that radiation damage was not the limiting factor in the tomography and by improving the sample environment higher resolution should be achievable with the same setup. At the same time, we conclude that without specific labeling single cell imaging in the hydrated state will remain somewhat limited, at least compared to other microscopy techniques. Reaching this sub-cellular resolution in extended tissue by region-of-interest tomography may become a unique application field for hard x-ray propagation imaging.

\section{SUMMARY AND OUTLOOK}

We have demonstrated the potential and the challenges in phase contrast tomography of unstained neuronal tissue. First, we have used a liquid metal microfocus laboratory source for phase contrast tomography of tadpole heads, thereby revealing the internal structure of the olfactory system on a micrometer resolution scale in the laboratory. The results have been obtained on a specimen prepared by critical point drying, 

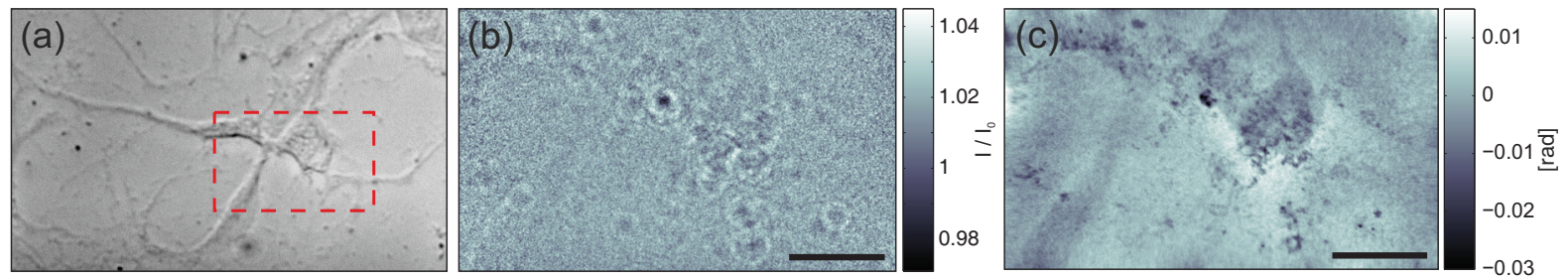

Figure 6. (Color online) (a) Optical micrograph of a mouse hippocampal neuron of primary cell culture. The area marked by the dashed rectangle corresponds to the area of the (b) phase contrast projection of the same cell. (c) CTF-retrieved phase obtained from 8 phase contrast projections for slightly different propagation distances. Scalebars denote $10 \mu \mathrm{m}$.

and are found to be very consistent with standard histology. The proof-of-concept shows that at least dried samples show sufficient contrast in this modality, and that internal structures can be revealed in fields of view which are relevant for applications. Importantly, time consuming and invasive sample preparation and the sequential imaging of hundreds to thousands of slices can be circumvented, paving the way to a throughput compatible with the statistical requirements of biomedical studies. By further improving the detector in the inverse geometry, a resolution below $1 \mu \mathrm{m}$ becomes achievable.

Second, we have used synchrotron radiation and a special x-ray waveguide optics providing optimized illumination and a resolution lower than $30 \mathrm{~nm}$, achieved for test patterns. ${ }^{27}$ We demonstrate the applicability of this technique to CNS tissue by providing 3D reconstructions, generated from CTF-based phase retrieval followed by filtered back projection. With the x-ray waveguide setup we also demonstrate the scalability of the approach by successfully reconstructing propagation based phase contrast projections of a thick hydrated brain slice that shows nearly no contrast in the direct contrast regime. This enables tomographic measurements resulting in a 3D representation of the internal structure in which individual neurons can be identified. We have shown that this technique has the potential to image the same structures even at a higher resolution, revealing sub-cellular features to be located inside a large block of surrounding tissue.

\section{Acknowledgments}

We thank Elisabeth Barski, Mihai Alevra and Sebastian Gliem for help in sample preparation. We also thank Michael Sprung for assistance during the beamtimes at P10. The work was supported by the excellence cluster 172 Molecular Physiology of the Brain/Microscopy at the Nanometer Range. Funding by the DFG collaborative research center SFB 755 'Nanoscale Photonic Imaging' and the German Ministry of Education and Research (BMBF-Verbundforschung, Grant No. 05K10MGA) is gratefully acknowledged.

\section{REFERENCES}

1. Pfeiffer, F., Kottler, C., Bunk, O., and David, C., "Hard X-Ray Phase Tomography with Low-Brilliance Sources," Phys. Rev. Lett. 98, 108105 (Mar 2007).

2. Zhu, P., Zhang, K., Wang, Z., Liu, Y., Liu, X., Wu, Z., McDonald, S. A., Marone, F., and Stampanoni, M., "Low-dose, simple, and fast grating-based x-ray phase-contrast imaging," PNAS 107(31), 1357613581 (2010).

3. Chien, C., Tseng, P., Chen, H., Hua, T., Chen, S., Chen, Y., Leng, W., Wang, C., Hwu, Y., Yin, G., Liang, K., Chen, F., Chu, Y., Yeh, H., Yang, Y., Yang, C., Zhang, G., Je, J., and Margaritondo, G., "Imaging cells and sub-cellular structures with ultrahigh resolution full-field x-ray microscopy," Biotechnol. Adv. 31(3), 375 - 386 (2013). High-Resolution multimodal imaging of living systems.

4. Thibault, P., Dierolf, M., Menzel, A., Bunk, O., David, C., and Pfeiffer, F., "High-Resolution Scanning X-ray Diffraction Microscopy," Science 321(5887), 379-382 (2008). 
5. Barty, A., Marchesini, S., Chapman, H. N., Cui, C., Howells, M. R., Shapiro, D. A., Minor, A. M., Spence, J. C. H., Weierstall, U., Ilavsky, J., Noy, A., Hau-Riege, S. P., Artyukhin, A. B., Baumann, T., Willey, T., Stolken, J., van Buuren, T., and Kinney, J. H., "Three-Dimensional Coherent X-Ray Diffraction Imaging of a Ceramic Nanofoam: Determination of Structural Deformation Mechanisms," Phys. Rev. Lett. 101, 055501 (July 2008).

6. Mayo, S., Davis, T., Gureyev, T., Miller, P., Paganin, D., Pogany, A., Stevenson, A., and Wilkins, S., "X-ray phase-contrast microscopy and microtomography," Opt. Express 11(19), 2289-2302 (2003).

7. Cloetens, P., Ludwig, W., Baruchel, J., Dyck, D. V., Landuyt, J. V., Guigay, J. P., and Schlenker, M., "Holotomography: Quantitative phase tomography with micrometer resolution using hard synchrotron radiation x rays," Appl. Phys. Lett. 75(19), 2912-2914 (1999).

8. Nugent, K. A., Gureyev, T. E., Cookson, D. F., Paganin, D., and Barnea, Z., "Quantitative Phase Imaging Using Hard X Rays," Phys. Rev. Lett. 77, 2961-2964 (Sep 1996).

9. Salditt, T. and Ducic, T., "X-ray microscopy for neuroscience: Novel opportunities by coherent optics," in [Super-Resolution Microscopy Techniques in the Neurosciences], Fornasiero, E. F. and Rizzoli, S. O., eds., Neuromethods 86, 257-290, Humana Press (2014).

10. Tuohimaa, T., Otendal, M., and Hertz, H. M., "Phase-contrast x-ray imaging with a liquid-metal-jetanode microfocus source," Appl. Phys. Lett. 91(7), 074104 (2007).

11. Bartels, M., Hernandez, V. H., Krenkel, M., Moser, T., and Salditt, T., "Phase contrast tomography of the mouse cochlea at microfocus x-ray sources," Appl. Phys. Lett. 103(8), 083703 (2013).

12. Walker, S. M., Schwyn, D. A., Mokso, R., Wicklein, M., Müller, T., Doube, M., Stampanoni, M., Krapp, H. G., and Taylor, G. K., "In Vivo time-resolved microtomography reveals the mechanics of the blowfly flight motor," PLoS Biol. 12, e1001823 (03 2014).

13. Goodman, J. W., [Introduction to Fourier Optics], Roberts \& Company: Englewood, Colorado (2005).

14. Witte, Y. D., Boone, M., Vlassenbroeck, J., Dierick, M., and Hoorebeke, L. V., "Bronnikov-aided correction for x-ray computed tomography," J. Opt. Soc. Am. A 26, 890-894 (Apr 2009).

15. Groso, A., Abela, R., and Stampanoni, M., "Implementation of a fast method for high resolution phase contrast tomography," Opt. Express 14, 8103-8110 (Sept. 2006).

16. Gabor, D., "A New Microscopic Principle," Nature 161, 777-778 (May 1948).

17. Turner, L., Dhal, B., Hayes, J., Mancuso, A., Nugent, K., Paterson, D., Scholten, R., Tran, C., and Peele, A., "X-ray phase imaging: Demonstration of extended conditions for homogeneous objects," Opt. Express 12, 2960-2965 (Jun 2004).

18. Gureyev, T. E., Davis, T. J., Pogany, A., Mayo, S. C., and Wilkins, S. W., "Optical phase retrieval by use of first born- and rytov-type approximations," Appl. Opt. 43, 2418-2430 (Apr 2004).

19. Cloetens, P., Contribution to Phase Contrast Imaging, Reconstruction and Tomography with Hard Synchrotron Radiation, PhD thesis, Vrije Universiteit Brussel (1999).

20. Guizar-Sicairos, M., Thurman, S. T., and Fienup, J. R., "Efficient subpixel image registration algorithms," Opt. Lett. 33, 156-158 (Jan. 2008).

21. Nieuwkoop, P. D. and Faber, J., "Normal table of Xenopus laevis (Daudin)," New York: Garland. (1994).

22. Nezlin, L. P. and Schild, D., "Individual olfactory sensory neurons project into more than one glomerulus in Xenopus laevis tadpole olfactory bulb," The Journal of Comparative Neurology 481(3), 233-239 (2005).

23. Olendrowitz, C., Bartels, M., Krenkel, M., Beerlink, A., Mokso, R., Sprung, M., and Salditt, T., "Phase-contrast x-ray imaging and tomography of the nematode Caenorhabditis elegans," Phys. Med. Biol. 57(16), 5309 (2012).

24. Otendal, M., Tuohimaa, T., Vogt, U., and Hertz, H. M., "A 9 keV electron-impact liquid-gallium-jet x-ray source," Rev. Sci. Instrum. 79(1), 016102 (2008).

25. Douissard, P.-A., Cecilia, A., Martin, T., Chevalier, V., Couchaud, M., Baumbach, T., Dupré, K., Kühbacher, M., and Rack, A., "A novel epitaxially grown LSO-based thin-film scintillator for microimaging using hard synchrotron radiation," J. Synchrotron Rad. 17, 571-583 (Sep 2010). 
26. Osterhoff, M. and Salditt, T., "Coherence filtering of x-ray waveguides: analytical and numerical approach," New Journal of Physics 13(10), 103026 (2011).

27. Krenkel, M., Bartels, M., and Salditt, T., "Transport of intensity phase reconstruction to solve the twin image problem in holographic x-ray imaging," Opt. Express 21, 2220-2235 (Jan. 2013). 